\title{
Functional Availability Analysis of Discrete Transport System Realized by SSF Simulator
}

\author{
Tomasz Walkowiak and Jacek Mazurkiewicz \\ Institute of Computer Engineering, Control and Robotics, \\ Wroclaw University of Technology, \\ ul. Janiszewskiego 11/17, 50-372 Wroclaw, Poland \\ \{Tomasz.Walkowiak, Jacek. Mazurkiewicz\}@pwr.wroc.pl
}

\begin{abstract}
The paper describes a novel approach to functional availability analysis of discrete transport systems realized using Scalable Simulation Framework (SSF). The proposed method is based on modeling and simulating of the system behavior by Monte Carlo simulation. No restriction on the system structure and on a kind of distribution is the main advantage of the method. The paper presents some exemplar system modeling. The authors stress the problem of influence of the functional parameters on final system availability. The problem described in the paper is practically essential for defining an organization of vehicle maintenance and transport system logistics.
\end{abstract}

\section{Introduction}

Decisions related to transport systems ought to be taken based on different and sometimes contradictory conditions. The transport systems are characterized by a very complex structure. The performance of the network can be impaired by various types of faults related to the transport vehicles, communication infrastructure or even by traffic congestion [8]. The analysis of transport system functionality can only be done if there is a formal model of the transport logistics. The classical models used for reliability analysis are mainly based on Markov or Semi-Markov processes [1] which are idealized and it is hard to reconcile them with practice. We suggest the Monte Carlo simulation 4 for proper functional parameters calculation. No restriction on the system structure and on a kind of distribution is the main advantage of the method 9. We propose to use the SSF (Scalable Simulation Framework) 2] instead of dedicated system elaboration. Our previous works [5], 7], [9], [10] show that it is very hard to prepare the simulator which includes all aspects of discrete transport. The SSF is a simulation core. It was developed for a usage in the SSFNet [3] a popular simulator of computer networks. We developed an extension to SSF allowing to simulate transport systems. We propose a formal model of discrete transport system to analyze functional aspects of complex systems. The presented in the next chapter discrete transport system model is based on the Polish Post regional centre of mail distribution.

M. Bubak et al. (Eds.): ICCS 2008, Part I, LNCS 5101, pp. 671 678, 2008.

(C) Springer-Verlag Berlin Heidelberg 2008 


\section{Discrete Transport System with Central Node and Time-Table (DTSCNTT)}

The model can be described as follows:

$$
D T S C N T T=\langle C N, N, R, V, I, M, T T\rangle,
$$

where: $C N$ - central node, $N$ - set of ordinary nodes, $R$ - set of routes, $V$ - set of vehicles, $I$ - input model, $M$ - set of maintenance crews and $T T$ - vehicles' time-table.

Commodities: We can discuss several kinds of a commodity transported in the system. Single kind commodity is placed in a unified container, and containers are transported by vehicles. The commodities are addressed and there are no other parameters describing them.

Nodes: We have single central node in the system. The central node is the destination of all commodities taken from other - ordinary nodes. Moreover the length between each two nodes is given.

Input Model: The aim of the system is to transport containers from the central node to ordinary nodes and in the opposite way. The containers are generated in each node. The central node is the global generator of commodities driven to each ordinary nodes of the system. The generation of containers is described by Poisson process. In case of central node there are separate processes for each ordinary node. Whereas, for ordinary nodes there is one process. The input model includes intensities of container generation in each ordinary node (routed to central node) and a table of intensities of containers for each ordinary node in the central node.

Vehicles: We assumed that all vehicles are of the same type and are described by following functional and reliability parameters: mean speed of a journey, capacity - number of containers which can be loaded, reliability function and time of vehicle maintenance. The central node is the start and destination of vehicle travels. The temporary state of each vehicle is characterized by following data: vehicle state, distance traveled from the begin of the route, capacity of the commodities. The vehicle running to the end of the route is able to take different kinds of commodity (located in unified containers, each container includes singlekind commodity). The vehicle hauling a commodity is always fully loaded or taking the last part of the commodity if it is less than its capacity.

Routes: Each route describes possible trip of vehicles. The set of routes we can describe as series of nodes:

$$
R=\left\langle c, v_{1}, \ldots, v_{n}, c\right\rangle \text { and } v_{i} \in N \text { and } c=C N .
$$

Maintenance Crews: Maintenance crews are identical and unrecognized. The crews are not assigned to any node, are not combined to any route, they operate in the whole system and are described only by the number of them. The temporary state of maintenance crews is characterized by: number of crews which are not involved into maintenance procedures and queue of vehicle waiting for the maintenance. 
Time-Table: Vehicles operate according to the time-table exactly as city buses or intercity coaches. The time-table consists of a set of routes (sequence of nodes staring and ending in the central node, times of approaching each node in the route and the recommended size of a vehicle. The number of used vehicle, or the capacity of vehicles does not depend on temporary situation described by number of transportation tasks or by the task amount for example. It means that it is possible to realize the journey by completely empty vehicle or the vehicle cannot load the available amount of commodity (the vehicle is to small). Time-table is a fixed element of the system in investigated time horizon, but it is possible to use different time-tables for different seasons or months of the year.

Each day a given time-table is realised, it means that at a time given by the time table a vehicle, selected randomly from vehicles available in the central node, departures from central node and loaded with containers addressed to each ordinary nodes included in a given route. This is done in a proportional way. Next, after arriving at given node (it takes some time according to vehicle speed - random process and road length) the vehicle waits in an input queue if there is any other vehicle being loaded/unload at the same time. There is only one handling point in each node. The time of loading/unloading vehicle is described by a random distribution. The containers addressed to given node are unloaded and empty space in the vehicle is filled by containers addressed to a central node. The operation is repeated in each node on the route and finally the vehicle is approaching the central node when is fully unloaded and after it is available for the next route. The process of vehicle operation could be stopped at any moment due to a failure (described by a random process). After the failure, the vehicle waits for a maintenance crew (if there are no available due to repairing other vehicles), is being repaired (random time) and after that it continues its journey.

\section{Simulation Methodology}

Discrete transport system described in the previous section is very hard to analyze by a formal model. It does not fit the Markov process framework. A common way of analyzing that kind of systems is a computer simulation. To analyze the system we must at first build a model and then operate the model. The system model needed for simulation has to encompass the system elements behavior and interaction between elements. In case of dependability we have to include system element reliability model. Except the system functionality model we have to model the traffic in the system. The data for simulation of a given real exemplar system consists of system element model (described in the system functionality meta-model formalism) and a given traffic configuration.

Once a model has been developed, it is executed on a computer by an eventsimulation, which is based on a idea of event. The event is described by time of event occurring, type of event (in case of DTSCNTT it could be vehicle failure) and element or set of elements of the system on which event has its influence. The simulation is done by analyzing a queue of event (sorted by time of event occurring) while updating the states of system elements according to rules related 
to a proper type of event. The event-simulation program could be written in general purpose programming language (like $\mathrm{C}++$ ), in fast prototyping environment (like Matlab) or special purpose discrete-event simulation kernels. One of such kernels, is the Scalable Simulation Framework (SSF) 2] which is a used for SSFNet 3] computer network simulator. SSF is an object-oriented API - a collection of class interfaces with prototype implementations. It is available in $\mathrm{C}++$ and Java. SSFAPI defines just five base classes: Entity, inChannel, outChannel, Process, and Event. The communication between entities and delivery of events is done by channels (channel mappings connects entities) 3]. For the purpose of simulating DTSCNTT we have used Parallel Real-time Immersive Modeling Environment (PRIME) 6] implementation of SSF due to much better documentation then that available for original SSF. We have developed a generic class (named DTSObject) derived from SSF Entity which is a base of classes modeling DTSCNTT objects like: scheduler, node, truck and crew which model the behavior of presented in section 2 discrete transport system. The effectiveness of simulation done in PRIME environment is very promising. The tests done on one batch of simulation of DTSCNTT exemplar described in the next section needed from 3.9 to 9 seconds on Pentium $2 \mathrm{GHz}$ computer. The time needed to perform one simulation depends on the number of events presented in the system, which is a result of DTSCNTT configuration. Due to a presence of randomness in the DTSCNTT model the analysis of it has to be done based on Monte-Carlo approach. It requires a large number of repeated simulation. The $\mathrm{SSF}$ is not a Monte-Carlo framework but by simple re-execution of the same code (of course we have to start from different values of random number seed) the statistical analysis of system behavior could be realized [12.

\section{Functional Availability of DTSCNTT}

The analysis of a given system requires a metric. We propose to use the availability of the system. We define it as an ability of realising the transportation task in required time. The availability is a probability measure. Introducing the following notation:

- $T$ - time measured from the moment when the container was introduced to the system to the moment when the container was transferred to the destination (random value),

- $T_{g}$ - guaranteed time of delivery, if exceeded the container is delayed,

$-N(t)$ - stochastic process describing the number of delayed containers at time $t$,

- $k$ - the level of acceptable delay,

we can define the functional availability $A_{k}(t)$ as a probability that the number of delayed containers at time $t$ does not exceed $k$, i.e.:

$$
A_{k}(t)=\operatorname{Pr}\{N(t) \leq k\}
$$

The calculation of stochastic process $N(t)$ is based on analysing a state of each 

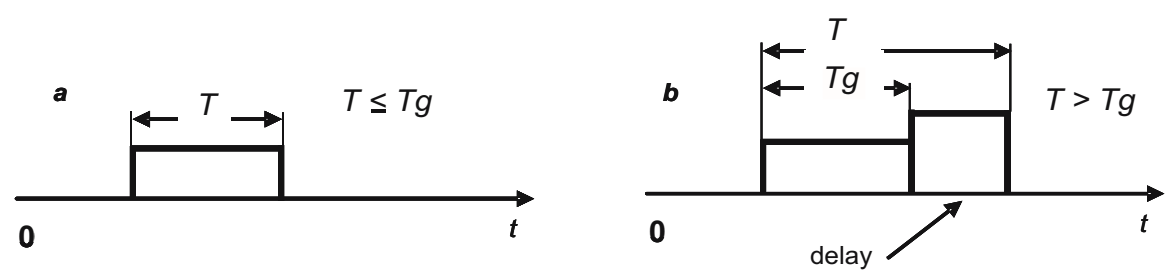

Fig. 1. The delivery in guaranteed time (a) and delayed delivery (b)

not yet delivered container. As illustrated in Fig. 1, we can observe two possible situations:

(a) - delivery was realised before guaranteed time $T_{g}$ - there is no delay,

(b) - delivery was delayed - time of delay: $T-T_{g}$.

\section{DTSCNTT Case Study}

For testing purposes of presented DTSCNTT system (chapter 2) and developed extension of SSF (chapter 3) we have developed an exemplar transport system. It consists of one central node (city Wroclaw, Poland) and three ordinary nodes (cites nearby Wroclaw: Rawicz, Olesnica and Nysa). The distances between nodes has been set approximating the real distances between used cities and they equal to: 85, 60 and $30 \mathrm{~km}$. We assumed a usage of 5 trucks (two with capacity set to 10 and three with capacity 15$)$ with mean speed $50 \mathrm{~km} / \mathrm{h}$. The vehicles realized 19 trips a day: from central node to ordinary node and the return trip. Failures of trucks were modeled by exponential distribution with mean time

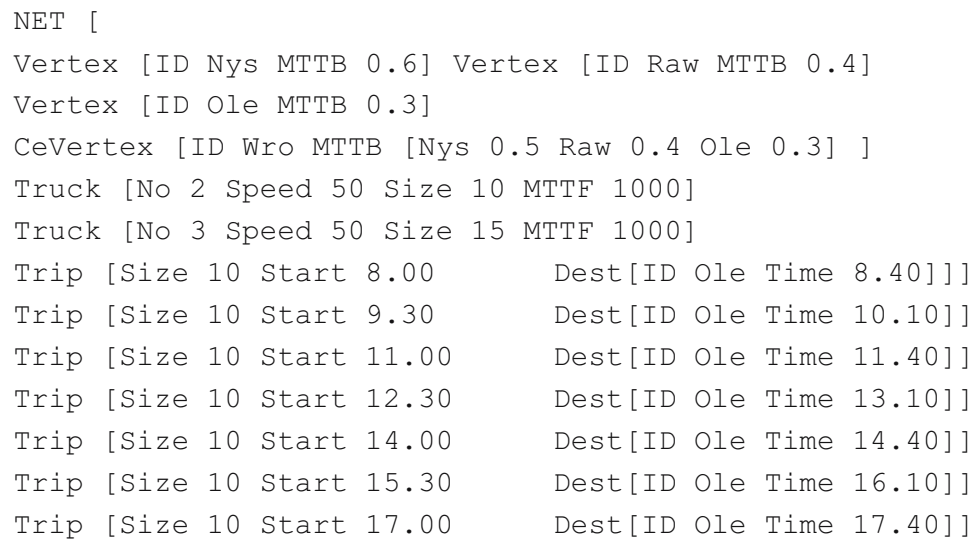

Fig. 2. Exemplar DTSCNTT description in DML file 


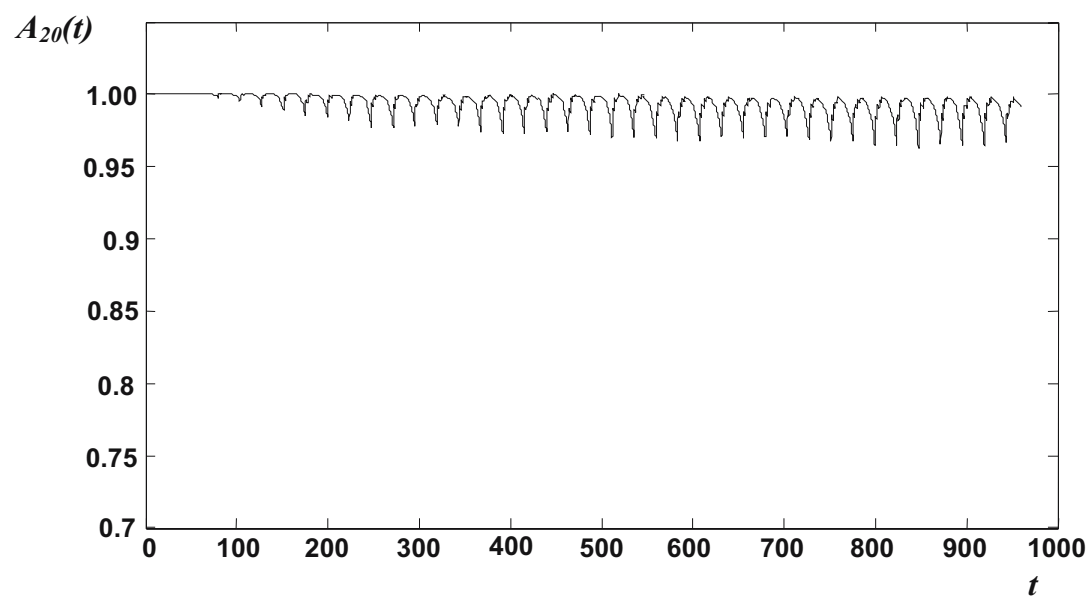

Fig. 3. Functional availability of the DTSCNTT, 5 trucks operate

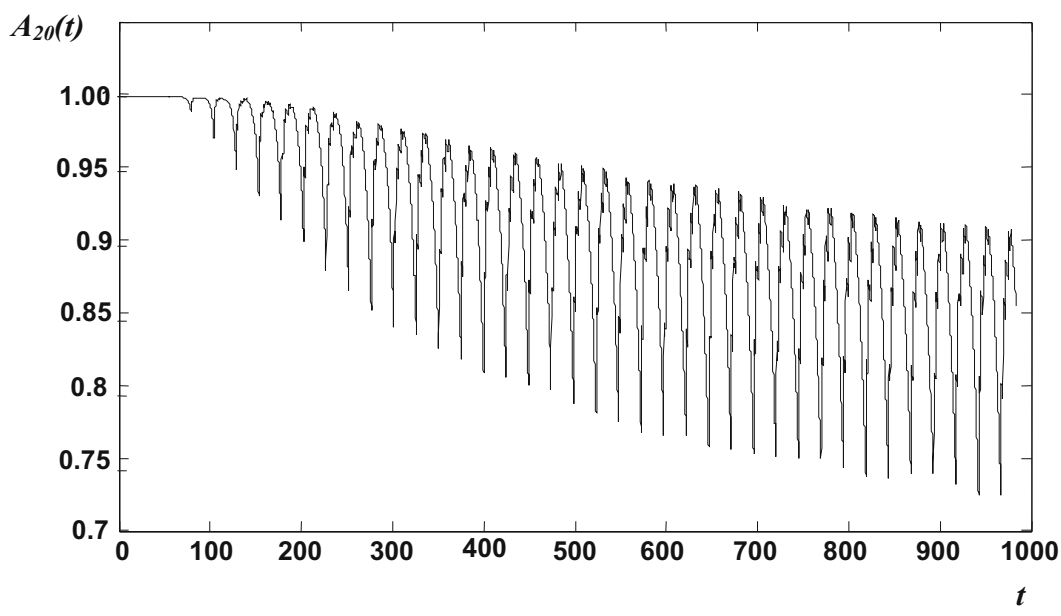

Fig. 4. Functional availability of the DTSCNTT, 4 trucks operate

to failure equal to $1000 \mathrm{~h}$. The repair time was modeled by normal distribution with mean value equal to $2 \mathrm{~h}$ and variance of $0.5 \mathrm{~h}$. The containers addressed to ordinary nodes were available in the central node at every $0.5,0.4$ and 0.3 of an hour respectively. Containers addressed to the central node were generated at every $0.6,0.4,0.3$ of hour in following ordinary nodes. There was a single maintenance crew. The availability of the system $A_{k}(t)$ was calculated with guaranteed time $T_{g}=24 \mathrm{~h}$ and parameter $k=20$. Time-table as well as other functional parameters were described in a DML file (see example in Fig. 2.). The Domain Modeling Language (DML) [6] is a SSF specific text-based language which 
includes a hierarchical list of attributes used to describe the topology of the model and model attributes values.

Based on 10000 time simulations (in each 100 days) the availability of system was calculated. Results presented in Fig. 3. shows the periodic changes. The situation is an effect of used time-tables and a method of cointainers' generation. The containers are generated during all day (by Poisson process) but according to a time-table trucks do not operate in the night. The probability of delay increases at the night, but selected number of trucks (5) is satisfactory for given system. We have also analyzed a system with a reduced number of vehicles (with 4). The resulting value of the availability function is presented in Fig. 4. It could be noticed that the availability of the system decreases due to lack of sufficient number of trucks. It should be noticed here that looking in the used time-table and not taking into consideration a randomness of the transport system (failures and traffic jams) only three vehicles should be enough to transport all the generated containers.

\section{Conclusion}

We have presented a simulation approach to functional analysis of Discrete Transport System with Central Node and Time-Table (DTSCNTT). The DTSCNTT models behavior of the Polish Post regional centre of mail distribution. Developed simulation software allows to analyze availability of the system in a function of all model parameters, like for example changes in a time-table or in a number of used trucks. Also, some economic analysis could be done following the idea presented in [5], [1], [12. It could be used for example for selection of the optimum value for SLA (service level agreement).

The presented results, i.e. changes of availability in a function of a number of used trucks shows that presented approach allows to answer a non trivial question what should be a number of vehicles to fulfill some requirements given to the transport system.

The implementation of DTSCNTT simulator done based on SSF allows to apply in a simple and fast way changes in the transport system model. Also the time performance of SSF kernel results in a very effective simulator of discrete transport system. Therefore, in our opinion introduced exemplar analysis shows that the described method of transport system modeling can serve for practical solving of essential decision problems related to an organization and parameters of a real transport system. The proposed analysis seems to be very useful for mail distribution centre organization.

Work reported in this paper was sponsored by a grant No. 4 T12C 05830 , (years: 2006-2009) from the Polish Committee for Scientific Research (KBN).

\section{References}

1. Barlow, R., Proschan, F.: Mathematical Theory of Reliability, Society for Industrial and Applied Mathematics, Philadelphia (1996)

2. Cowie, J.H.: Scalable Simulation Framework API reference manual (1999), http://www.ssfnet.org/SSFdocs/ssfapiManual.pdf 
3. Cowie, J.H., Nicol, D.M., Ogielski, A.T.: Modeling the Global Internet. Computing in Science and Engineering 1(1), 42-50 (1999)

4. Fishman: Monte Carlo: Concepts, Algorithms, and Applications. Springer-Verlag, New York (1996)

5. Kaplon, K., Mazurkiewicz, J., Walkowiak, T.: Economic Analysis of Discrete Transport Systems. Risk Decision and Policy 8(3), 179-190 (2003)

6. Liu, J.: Parallel Real-time Immersive Modeling Environment (PRIME), Scalable Simulation Framework (SSF), User's manual, Colorado School of Mines Dep. of Mathematical and Computer Sciences (2006), http://prime.mines.edu

7. Mazurkiewicz, J., Walkowiak, T.: Fuzzy Economic Analysis of Simulated Discrete Transport System. In: Rutkowski, L., Siekmann, J.H., Tadeusiewicz, R., Zadeh, L.A. (eds.) ICAISC 2004. LNCS (LNAI), vol. 3070, pp. 1161-1167. Springer, Heidelberg (2004)

8. Sanso, B., Milot, L.: Performability of a Congested Urban-Transportation Network when Accident Information is Available. Transportation Science 33, 1 (1999)

9. Walkowiak, T., Mazurkiewicz, J.: Hybrid Approach to Reliability and Functional Analysis of Discrete Transport System. In: Bubak, M., van Albada, G.D., Sloot, P.M.A., Dongarra, J. (eds.) ICCS 2004. LNCS, vol. 3037, pp. 236-243. Springer, Heidelberg (2004)

10. Walkowiak, T., Mazurkiewicz, J.: Reliability and Functional Analysis of Discrete Transport System with Dispatcher. In: Advances in Safety and Reliability, European Safety and Reliability Conference - ESREL 2005, pp. 2017-2023. Taylor \& Francis Group, London (2005)

11. Walkowiak, T., Mazurkiewicz, J.: Simulation Based Management and Risk Analysis of Discrete Transport Systems. In: IEEE TEHOSS 2005 Conference, pp. 431-436 (2005)

12. Walkowiak, T., Mazurkiewicz, J.: Discrete transport system simulated by SSF for reliability and functional analysis. In: International Conference on Dependability of Computer Systems. DepCoS - RELCOMEX 2007, pp. 352-359. IEEE Computer Society Press, Los Alamitos (2007) 